\title{
A Meta-Comparison of the Effects of High-Intensity Interval Training to Those of Small-Sided Games and Other Training Protocols on Parameters Related to the Physiology and Performance of Youth Soccer Players
}

Philipp Kunz ${ }^{*^{*}}$, Florian Azad Engel ${ }^{2}$, Hans-Christer Holmberg ${ }^{3,4}$ and Billy Sperlich ${ }^{1}$

\begin{abstract}
Background: High-intensity interval training (HIIT) is frequently employed to improve the endurance of various types of athletes. To determine whether youth soccer players may benefit from the intermittent load and time efficiency of HIIT, we performed a meta-analysis of the relevant scientific literature.

Objectives: Our primary objective was to compare changes in various physiological parameters related to the performance of youth soccer players in response to running-based HIIT to the effects of other common training protocols (i.e., small-sided games, technical training and soccer-specific training, or high-volume endurance training). A secondary objective was to compare specifically running-based HIIT to a soccer-specific form of HIIT known as small-sided games (SSG) in this same respect, since this latter type of training is being discussed extensively by coaches.

Method: A systematic search of the PubMed, SPORTDiscus, and Web of Science databases was performed in August of 2017 and updated during the review process in December of 2018. The criteria for inclusion of articles for analysis were as follows: (1) comparison of HIIT to SSG or some other training protocol employing a pre-post design, (2) involvement of healthy young athletes ( $\leq 18$ years old), and (3) assessment of variables related to endurance or soccer performance. Hedges' $g$ effect size $\left(d_{\mathrm{ppc} 2}\right)$ and associated $95 \%$ confidence intervals for the comparison of the responses to HIIT and other interventions were calculated.

(Continued on next page)
\end{abstract}

\footnotetext{
* Correspondence: philipp.kunz@uni-wuerzburg.de

${ }^{1}$ Integrative \& Experimental Exercise Science and Training, Institute of Sport

Science, University of Würzburg, Judenbühlweg 11, 97082 Würzburg,

Germany

Full list of author information is available at the end of the article
} 
(Continued from previous page)

Results: Nine studies, involving 232 young soccer players (mean age 16.2 \pm 1.6 years), were examined. Endurance training in the form of HIIT or SSG produced similar positive effects on most parameters assessed, including peak oxygen uptake and maximal running performance during incremental running (expressed as $V_{\text {max }}$ or maximal aerobic speed (MAS)), shuttle runs (expressed as the distance covered or time to exhaustion), and time-trials, as well as submaximal variables such as running economy and running velocity at the lactate threshold. HIIT induced a moderate improvement in soccer-related tests involving technical exercises with the soccer ball and other game-specific parameters (i.e., total distance covered, number of sprints, and number of involvements with the ball). Neuromuscular parameters were largely unaffected by HIIT or SSG.

Conclusion: The present meta-analysis indicates that HIIT and SSG have equally beneficial impacts on variables related to the endurance and soccer-specific performance of youth soccer players, but little influence on neuromuscular performance.

Keywords: Adolescents, Children, Conditioning, Endurance, Repeated sprint

\section{Key Points}

- Various reports on responses of both youth and adults to high-intensity interval training (HIIT) have been published, but no systematic comparison of the effects of HIIT and other training regimes on parameters related to the performance of youth soccer players has yet been published.

- The calculated effect sizes indicate that HIIT has more beneficial effects than various other exercise programs on all of the parameters examined, with the exceptions of sprinting and jumping performance and repeated sprint ability.

- Small-sided games (SSG), a sport-specific form of HIIT, exerted similar effects on parameters related to soccer performance (i.e., maximal oxygen uptake, maximal running performance, running economy, and running at the lactate threshold).

- HIIT and SSG both enhance the key performance of youth soccer players in a time-efficient manner, leaving more time for training sport-specific skills such as technique and tactics.

\section{Background}

Youth soccer is characterized by constant changes in the intensity of a variety of activities, including standing, walking, running, and sprinting with frequent changes in direction, as well as jumping, often with involvement of the ball and/or opponents [1]. This varying intensity (from low to high), as well as matches lasting as long as 90 min (depending on age), involves on average $>80 \%$ of peak heart rate [2] and approximately $75 \%$ of maximal oxygen uptake [1] in youth soccer players, imposing considerable demands on both aerobic and anaerobic energy production (i.e., in connection with short sprints, jumps, tackles) [1, 3]. Accordingly, high-level endurance performance is an important prerequisite for success [3].
High-volume or continuous low-intensity training has been employed successfully to improve certain aspects of endurance performance, such as peak oxygen uptake $\left(\mathrm{VO}_{2 \text { peak }}\right)$, individual anaerobic threshold, and/or maximal velocity in youth soccer players $[4,5]$. HIIT, characterized by periods of intense exercise at $>90 \%$ of peak heart rate $\left(\mathrm{HR}_{\text {peak }}\right)$ alternating with periods of low-intensity activity [6], and SSG, a soccer-specific form of HIIT [7], show great potential to improve certain aspects of the endurance of youth soccer players. Both of these types of training improve key variables related to success in soccer, e.g., $\mathrm{VO}_{2 \text { peak }}[8,9]$ and maximal running performance (i.e., shuttle run testing and time-trials) $[10,11]$, as well as performance in connection with soccer-specific tests (i.e., total distance covered, number of sprints, and number of contacts with the ball) $[12,13]$ in a time-efficient manner [14].

The lengths of training periods and intervals of rest, number of intervals and sessions of HIIT or SSG per week, and the number of players and size of the pitch can be varied in virtually an endless number of ways [14-18]. Various HIIT protocols improve different indicators of endurance performance [10] and SSG enhances both fitness and soccer-specific performance [14, 16]. Clearly, in addition to technical and tactical skills, such physiological factors are also important determinants of success [19].

From a practical point of view, questions arise among coaches concerning (1) the overall responses of youth soccer players to HIIT in comparison to other endurance protocols with respect to endurance and other important determinants of performance, such as the ability to sprint and jump; and (2) whether SSG, a sport-specific form of HIIT, results in improvement of these same parameters comparable to those achieved with running-based HIIT.

Our aim here was to systematically compare the effects of HIIT on variables related to the performance by youth 
soccer players as reported in numerous publications during the past decade to those of other training regimes (in particular SSG). Such analysis should aid in designing HIIT and/or SSG to improve the endurance of youth soccer players most effectively.

\section{Methods}

\section{Databases and Search Profile}

This systematic analysis of peer-reviewed investigations on the effects of HIIT and SSG on several parameters related to performance in youth soccer players was conducted in accordance with established guidelines [20]. A comprehensive computerized search of the PubMed, SPORTDiscus, and Web of Science databases, with no restriction as to year of publication, was performed in August of 2017, with an update during the review process in December of 2018, employing the following search strings: high-intensity interval training OR high-intensity training OR intensive interval training OR aerobic interval training OR sprint interval training OR specific endurance training OR aerobic endurance OR aerobic training AND young players OR young athletes OR adolescent athletes OR teen athletes OR junior athletes OR children athletes OR children OR adolescents AND soccer OR football.

The search was limited to original research articles written in English and published in peer-reviewed journals. The screening and selection process is illustrated in Fig. 1.

In addition, the references cited by the articles retrieved were examined for potential relevance. Data was extracted from the studies included by one of the authors and this extraction checked independently by a second author.

\section{Selection and Quality Assessment of Articles}

The inclusion criteria were as follows: (1) involvement of endurance training in the form of HIIT (e.g., at $\geq 90 \%$ of maximal oxygen uptake [21], $90-95 \%$ of $\mathrm{HR}_{\text {peak }}$ [10], or as (supra)maximal interval sprinting [22]); (2) involvement of male soccer players 18 years of age or younger; (3) pre- and post-assessment of exercise performance, physiological parameters related to performance, and/or soccer-specific performance; (4) intervention for at least 4 weeks; and (5) inclusion of a control treatment (i.e., SSG, high-volume training, or technical training).

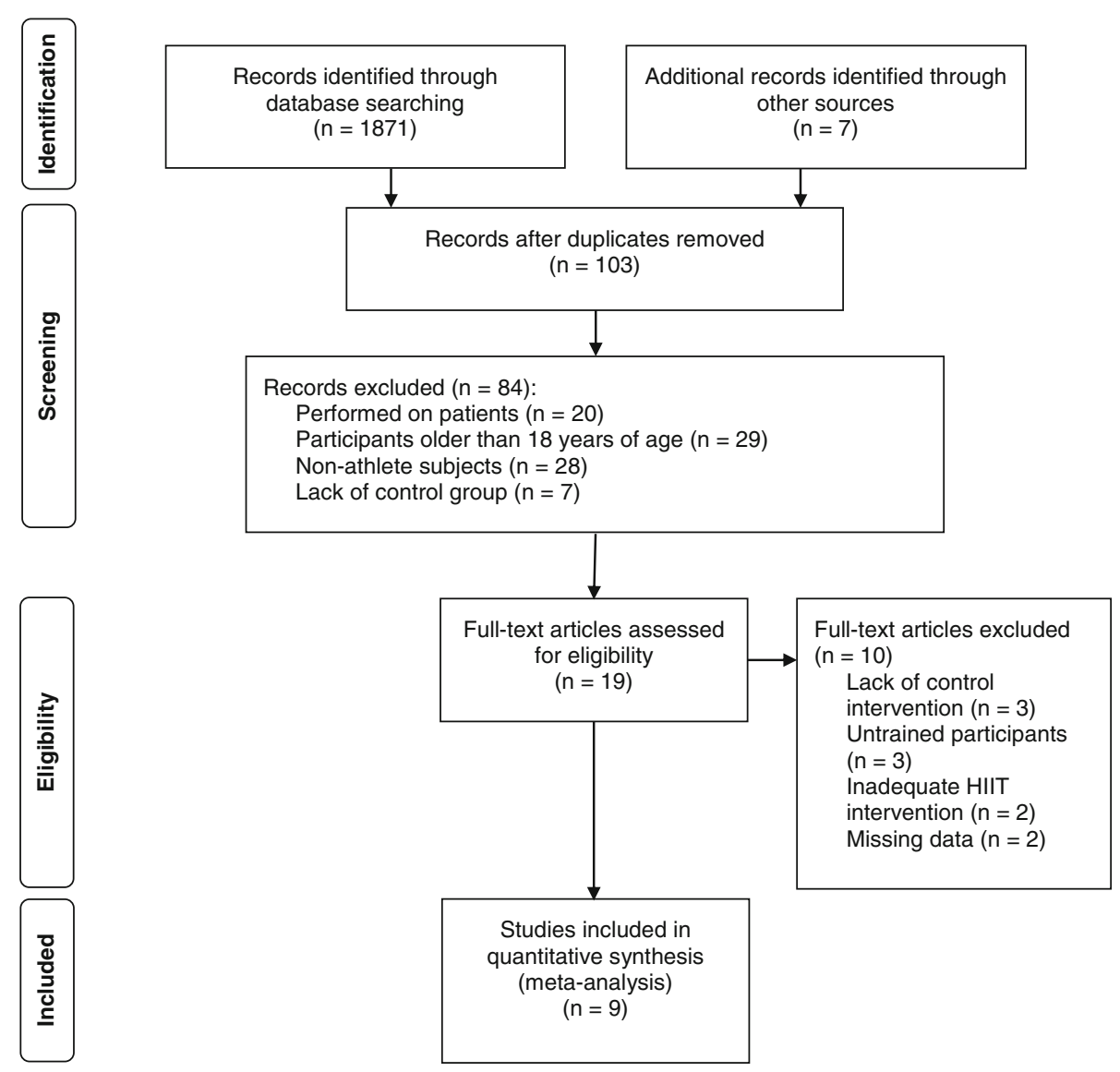

Fig. 1 Selection of the articles to be analyzed, from initial identification to inclusion 
Studies on patients with specific conditions (e.g., obesity, diabetes mellitus, or asthma) were excluded, as were conference abstracts, dissertations, theses, and articles that had not undergone peer review.

To ensure the methodological quality of the articles selected, the criteria of the Physiotherapy Evidence Database (PEDro) scale were applied, with one point for each criterion fulfilled and a maximal possible score of 10 [23]. The quality of each article is documented in Table 1.

\section{Statistical Analyses}

The findings on HIIT were compared to those with (1) all of the other interventions, (2) all of the other interventions except SSG, and (3) SSG alone.

Effect sizes (ES) were calculated as the mean difference between the experimental and control groups, divided by $\mathrm{SD}_{\text {pooled }}$, as recommended by Carlson and Schmidt [24].

$$
\text { SDpooled }=\sqrt{\frac{\left(n_{\text {exp }}-1\right) \mathrm{SD}_{\exp }^{2}+\left(n_{\text {contr }}-1\right) \mathrm{SD}_{\text {contr }}^{2}}{n_{\text {exp }}+n_{\text {contr }}-2}}
$$

where $n$ is the number of participants in the experimental $\left(n_{\text {exp }}\right)$ and control groups $\left(n_{\text {cont }}\right)$ and $\mathrm{SD}_{\text {exp }}$ and $\mathrm{SD}_{\text {cont }}$ the corresponding pretest standard deviations.

In addition, in light of the tendency for findings with small samples to be positively biased, leading to overestimation, a correction factor $(\mathrm{CP})$ was employed:

$$
\mathrm{CP}=1-\frac{3}{4 \times\left(n_{\exp }+n_{\text {contr }}-2\right)-1}
$$

This approach to the calculation of ES $\left(d_{\mathrm{ppc} 2}\right)$ is optimal in the case of studies involving pre- and post-testing and a control group [25]:

Table 1 The Physiotherapy Evidence Database (PEDro) score for each article included

\begin{tabular}{lllllllllllll}
\hline & \multicolumn{1}{c}{ Item } & & & & & & & & & \\
\cline { 2 - 10 } Article & $1^{\text {a }}$ & 2 & 3 & 4 & 5 & 6 & 7 & 8 & 9 & 10 & 11 & Total \\
\hline Los Arcos et al. (2015) [27] & 1 & 1 & 1 & 1 & 0 & 0 & 0 & 1 & 1 & 1 & 1 & 7 \\
Faude et al. (2013) [4] & 1 & 1 & 1 & 1 & 0 & 0 & 0 & 0 & 1 & 1 & 1 & 6 \\
Faude et al. (2014) [29] & 1 & 1 & 1 & 1 & 0 & 0 & 0 & 0 & 1 & 1 & 1 & 6 \\
Helgerud et al. (2001) [29] & 1 & 1 & 1 & 1 & 0 & 0 & 0 & 1 & 1 & 1 & 1 & 7 \\
Hill-Haas et al. (2009) [11] & 1 & 1 & 1 & 1 & 0 & 0 & 0 & 0 & 1 & 1 & 1 & 6 \\
Impellizzeri et al. (2008) [12] & 1 & 1 & 1 & 1 & 0 & 0 & 0 & 1 & 1 & 1 & 1 & 7 \\
Impellizzeri et al. (2006) [28] & 1 & 1 & 1 & 1 & 0 & 0 & 0 & 0 & 1 & 1 & 1 & 6 \\
Siegler et al. (2013) [30] & 1 & 0 & 0 & 1 & 0 & 0 & 0 & 1 & 1 & 1 & 1 & 5 \\
Sperlich et al. (2011) [10] & 1 & 1 & 1 & 1 & 0 & 0 & 0 & 1 & 1 & 1 & 1 & 7 \\
\hline
\end{tabular}

${ }^{\mathrm{a}}$ This item was not included when calculating the PEDro score $d_{\mathrm{ppc} 2}=\mathrm{CP}\left[\frac{\left(M_{\text {post, } \exp }-M_{\text {pre, exp }}\right)-\left(M_{\text {post }, \text { contr }}-M_{\text {pre,contr }}\right)}{\mathrm{SD}_{\text {pooled }}}\right.$

The ES values obtained were classified as trivial $(<0.10)$, small $(0.10-0.30)$, moderate $(0.30-0.50)$, or large $(>0.50)$ [26]. Heterogeneity was assessed using an $I^{2}$ value and the 95\% confidence interval (CI) calculated.

All statistical analyses were carried out in version 11.5.1.0 of the Med-Calc software (MedCalc Software, Mariakerke, Belgium).

\section{Results}

\section{Characteristics of the Studies Analyzed}

Nine studies were included (see Table 2 for a summary) and assessed in accordance with the PEDro scale, resulting in an average score of 6.3 (range 5-7). In four of these, SSG was the control program [11, 27-29], the five others comparing either a form of high-volume training $[4,10,30]$ or technical soccer training $[12,13]$ to HIIT.

Altogether, these studies involved 232 male soccer players (mean 22.6 \pm 8.2 participants per study; range $15-39$ ), $13-18$ years of age (mean age: $16.2 \pm 1.6$ years), of whom $50 \%$ performed intense interval training and the other $50 \%$ control programs of exercise, i.e., either SSG, some form of low-intensity high-volume exercise, or soccer-specific drills (e.g., generic soccer training, technical and tactical training).

All of these participants played regularly for a club, in most cases professional. Four studies [11, 12, 27, 28] explicitly excluded goalkeepers, with their special physical requirements and training regimes, from their interventions. In five of these nine studies in which this parameter was measured $[10-13,28]$, the initial mean $\mathrm{VO}_{2 \text { peak }}$ of the subjects was $57.4 \pm 1.7$ (range 55.1-60.2) $\mathrm{mL} \cdot \mathrm{kg}^{-1} \cdot \mathrm{min}^{-1} . \mathrm{HR}_{\max }$ was determined with incremental treadmill tests $[10-13,28]$, incremental field tests [4, 29], or the University of Montreal Test (UM-TT) [27].

\section{Protocols of the HIIT Interventions}

The mean duration of the interventions was $6.4 \pm 2.0$ weeks (range 4-10), with an average of $2.9 \pm 0.9$ sessions per week (range 2-4.5).

The HIIT protocols employed differed with respect to intensity, duration, and work-to-rest ratio (Table 2). Notably, three studies involved $4 \times 4 \mathrm{~min}$ of running at 90 $95 \%$ of maximal heart rate $\left(\mathrm{HR}_{\max }\right)$, with 3 -min intervals of rest $[12,13,28]$.

In most cases, the target intensity was $>90 \% \mathrm{HR}_{\max }$ $[10,12,27,28,30]$, although two studies set this intensity at $25-40 \%$ greater than the participant's individual anaerobic threshold velocity $[4,29]$. Two studies employed elements of functional HIIT, such as repeated squats, jumps, interval sprints, and agility drills $[11,30]$. The intervals of exercise were either short $(\sim 15-30 \mathrm{~s}$; $[4,29]$ or 


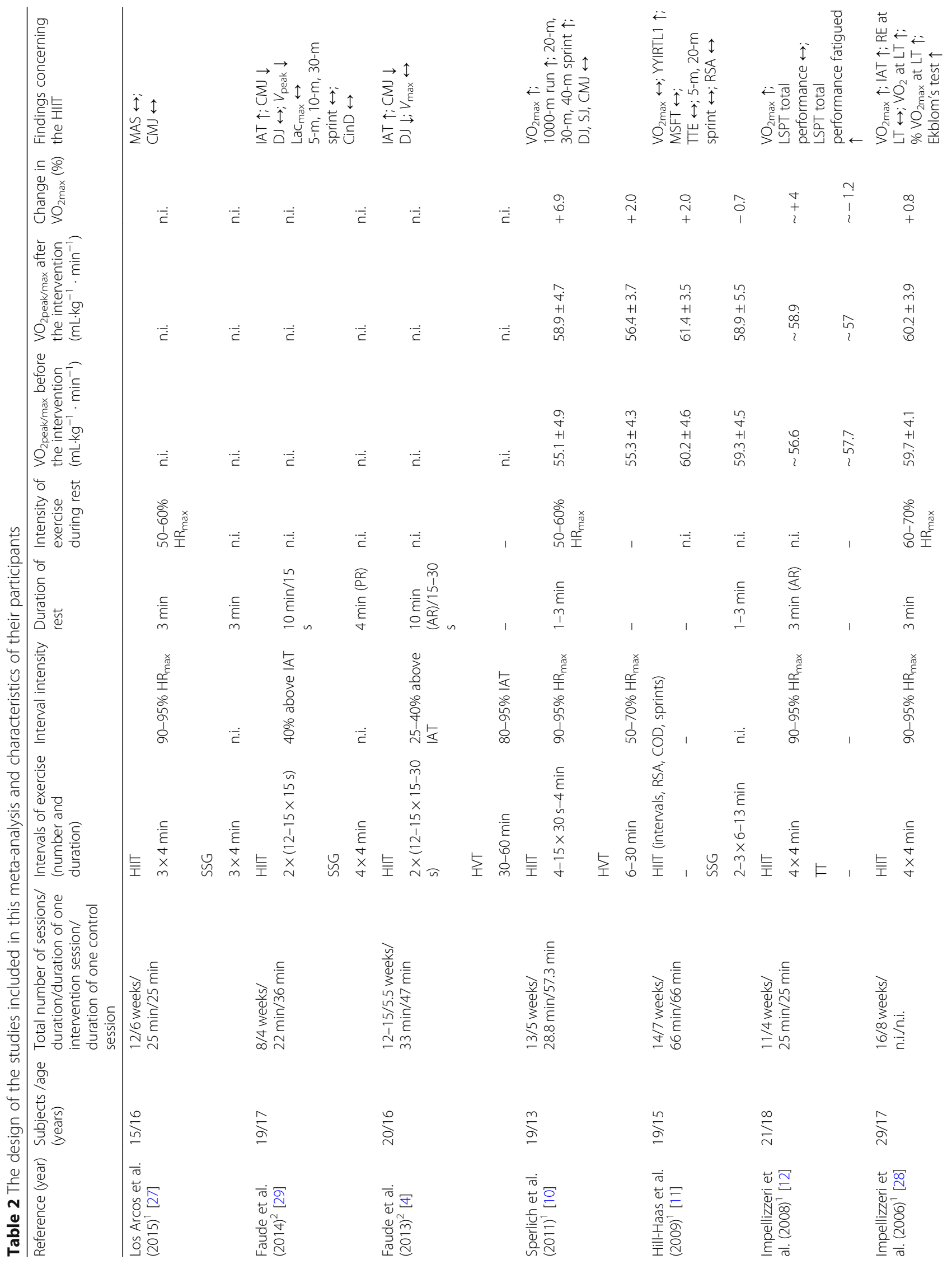


Kun et al. Sports Medicine - Open $\quad$ (2019) 5:7

Page 6 of 13

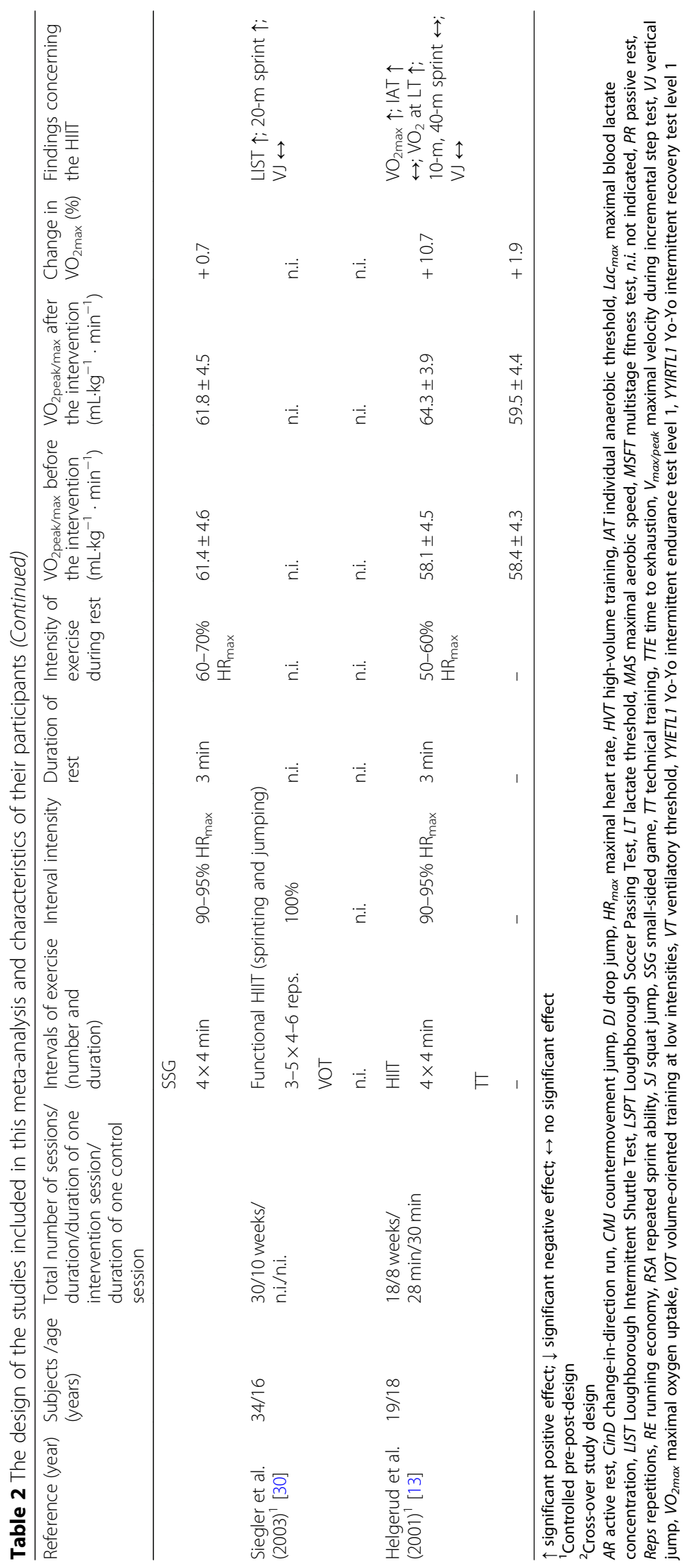


4 min in length $[12,13,27,28]$, with one study involving a mixture (30 s-4 $\mathrm{min})$ [10]. The recovery period between intervals of exercise ranged from 15 to $180 \mathrm{~s}$.

\section{The SSG Interventions}

In four cases, HIIT was compared to SSG [11, 27-29]. Two of these studies employed 4 $\times 4$-min games [28, 29], one $3 \times 4-\mathrm{min}$ of SSG [27], and one 2-3 sets of 613-min games [11]. The recovery between intervals of exercise ranged from 1 to $4 \mathrm{~min}$ and the pitch dimensions varied, as did the duration and number of players (range 2 against 1 to 7 against 7).

\section{Maximal or Peak Oxygen Uptake Comparison of HIIT to All Other Interventions}

In comparison to all other interventions, HIIT induced a moderate mean positive effect on peak oxygen uptake (mean $d_{\mathrm{ppc} 2} 0.45 \pm 0.46$; range 0.08-1.11). Among the four analyses of peak or maximal oxygen uptake, one effect was trivial positive [28], one small positive [11], one moderate positive [10], and one large positive [13] (Fig. 2).

\section{Comparison of HIIT to All Other Interventions Except SSG}

In comparison to all interventions except SSG, HIT resulted in a large mean positive effect on maximal or peak oxygen uptake (mean $d_{\mathrm{ppc} 2} 0.75 \pm 0.50$; range 0.40 -
1.11). In the two studies analyzed in this respect, one reported a moderate positive [10] and the other a large positive effect [13] (Fig. 3).

\section{Comparison of HIIT to SSG}

In comparison to SSG, HIT exerted a small positive effect on maximal or peak oxygen uptake (mean $d_{\mathrm{ppc} 2}$ $0.15 \pm 0.10$; range $0.08-0.22$ ), as shown by two studies, one demonstrating a trivial positive effect [28] and the other a small positive effect [11] (Fig. 4).

\section{Effects on Parameters Related to Maximal Running Performance HIIT Versus All Other Interventions}

As assessed in six studies, HIIT induced a moderate mean positive effect on parameters related to maximal running performance (mean $d_{\mathrm{ppc} 2} 0.30 \pm 0.58$; range $0.78-1.34)[4,10,11,27,29,30]$, in association with either incremental running tests (expressed as maximal aerobic speed (MAS) or $V_{\max }$ ) [4, 27, 29], different shuttle run tests $[11,30]$, or a $1000-\mathrm{m}$ time-trial run [10] (Fig. 2). The $I^{2}$ value of $14 \%$ indicated low heterogeneity.

\section{HIIT Versus All Other Interventions Except SSG}

As examined in three studies [4, 10,30], in comparison to all other interventions except SSG, HIIT induced a

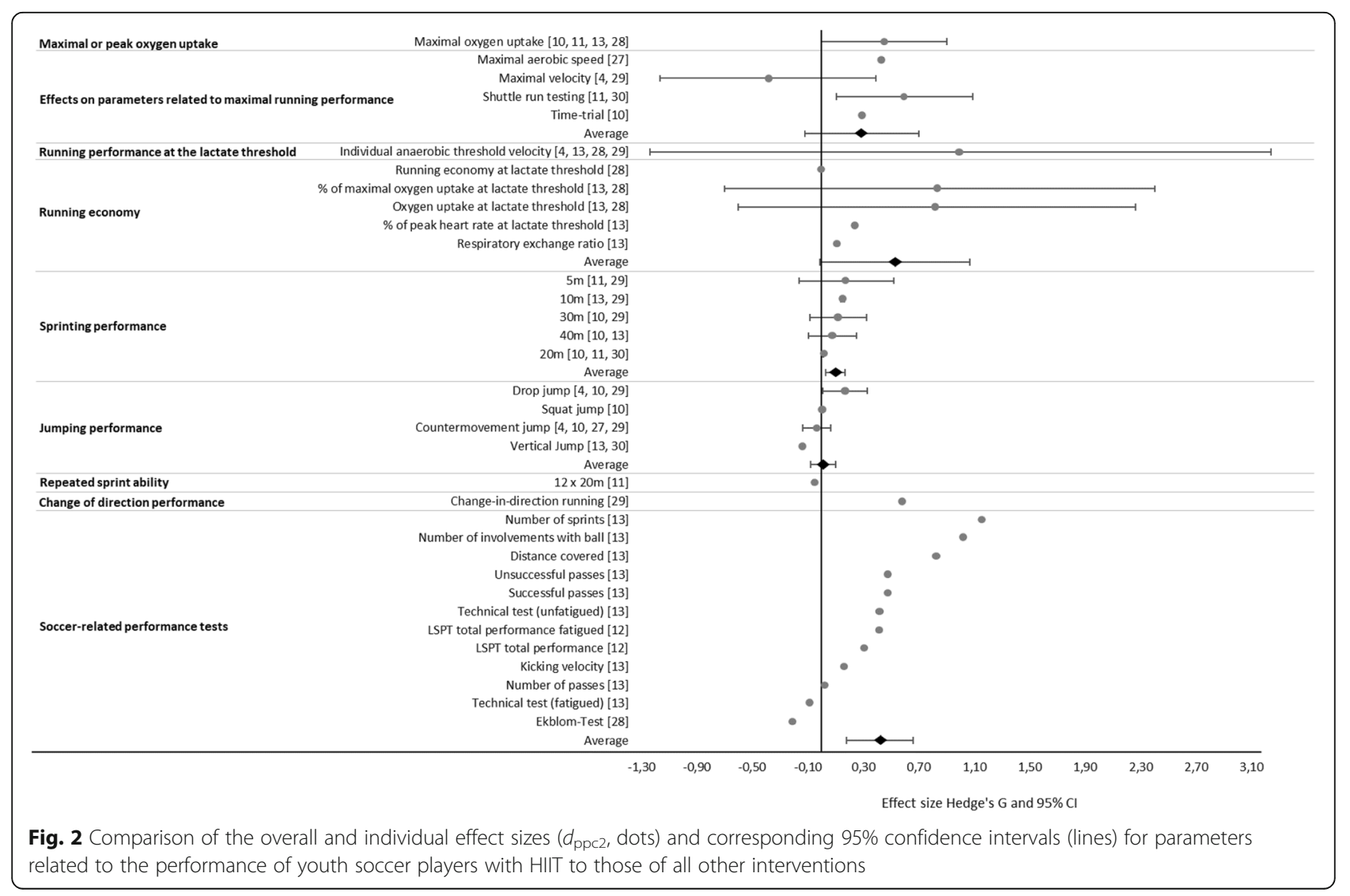




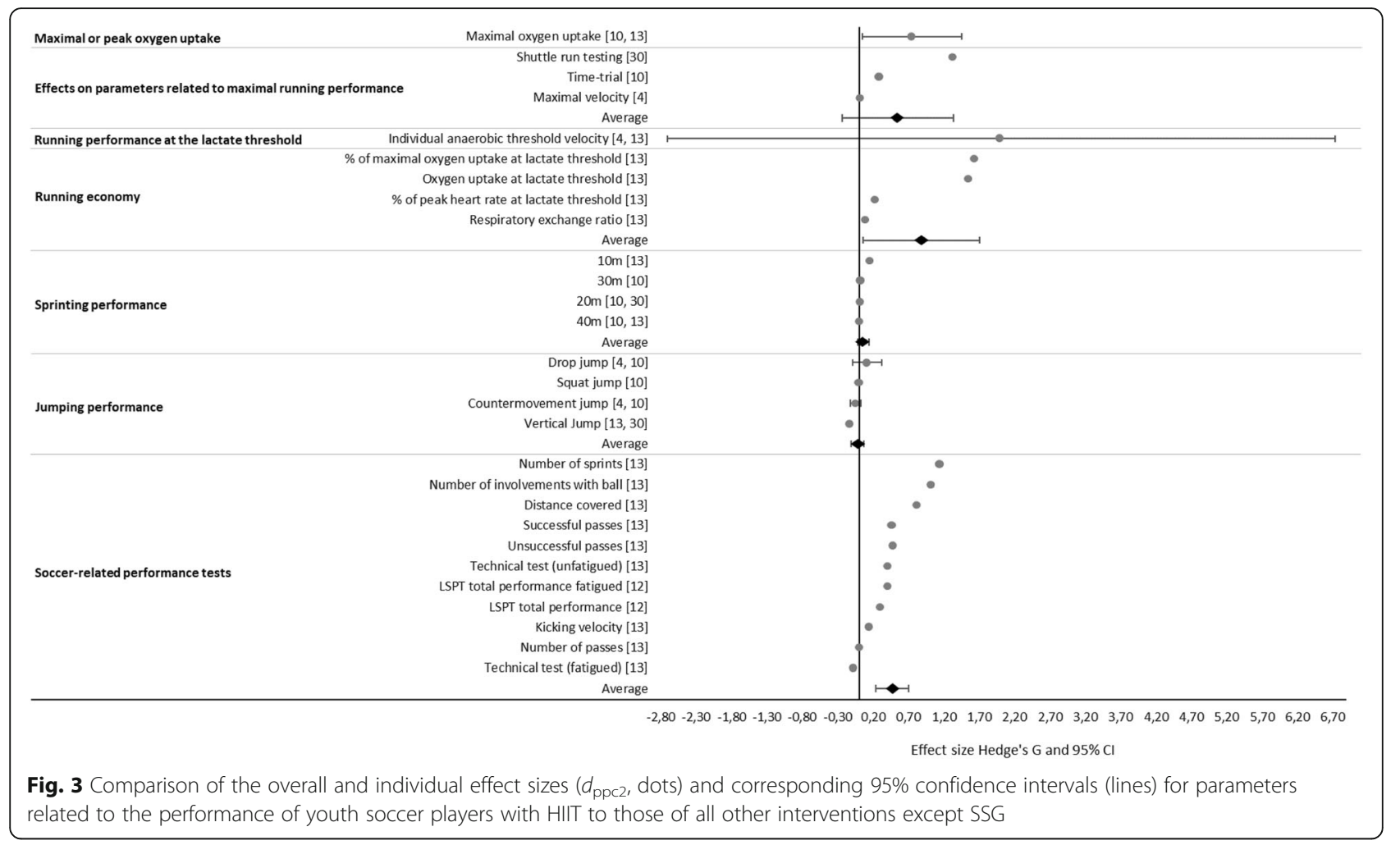

large positive mean effect on maximal running performance (mean $d_{\mathrm{ppc} 2} 0.55 \pm 0.70$; range 0.01-1.34) (Fig. 3).

\section{HIIT Versus SSG}

Three studies [11, 27, 29] demonstrated small positive effects of HIIT on maximal running performance (mean $d_{\mathrm{ppc} 2} 0.14 \pm 0.52$; range $\left.-0.78-0.45\right)$ in comparison to
SSG, as determined by incremental running tests [27, 29] and shuttle run testing [11] (Fig. 4).

\section{Running Performance at the Lactate Threshold HIIT Versus All Other Interventions}

With respect to running performance at the lactate threshold, reported in four cases, HIT exerted a large positive mean effect in comparison to all other

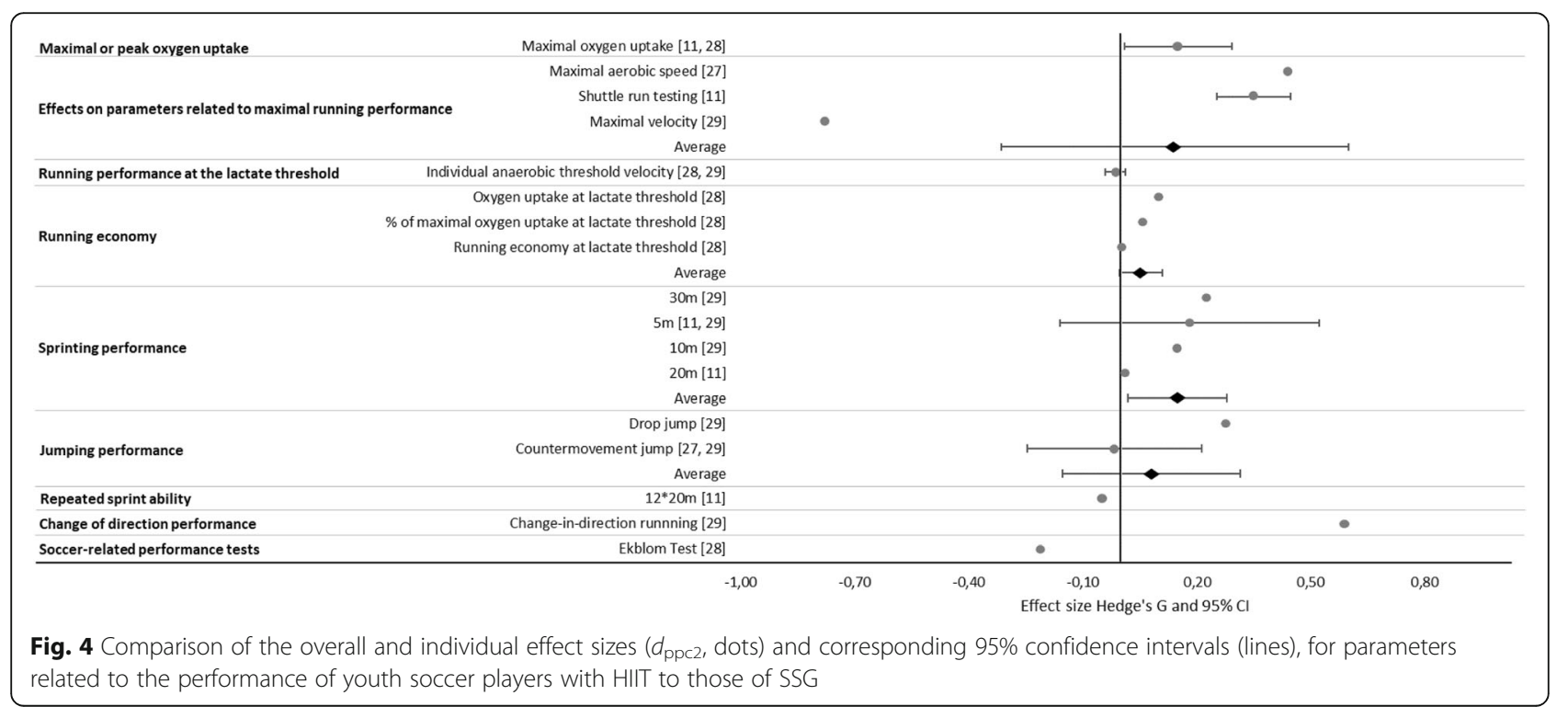


interventions (mean $d_{\mathrm{ppc} 2} 1.00 \pm 2.29$; range $-0.40-$ 4.42). One of these four studies indicated a moderate negative [4], one trivial negative [28], one no [29], and one large positive effect [13] (Fig. 2). The lactate threshold was determined either from the lactate curve $[4,29]$ according to the procedure of Stegmann and colleagues [31] or defined as a blood lactate concentration 1.5 $\mathrm{mmol} / \mathrm{L}$ higher than baseline $[13,28]$.

\section{HIIT Versus All Other Interventions Except SSG}

As shown in two reports, in comparison to all other interventions except SSG, HIIT had a large positive effect on running performance at the lactate threshold (mean $d_{\mathrm{ppc} 2} 2.01 \pm 3.41$; range $-0.40-4.42$ ) [4, 13] (Fig. 3).

\section{HIIT Versus SSG}

Comparison of HIIT to SSG in two publications $[28,29]$ demonstrated a trivial negative mean effect of the former on running performance at the lactate threshold (mean $d_{\mathrm{ppc} 2}-0.01 \pm 0.02$; range $-0.03-0.00$ ) (Fig. 4).

\section{Running Economy HIIT Versus All Other Interventions}

In comparison to all other interventions, HIIT induced a large positive mean effect on running economy (mean $d_{\mathrm{ppc} 2} 0.53 \pm 0.74 ;$ range $0.00-1.64$ ). Two trivial positive [28], three small positive [13, 28], and two large positive effects [13] were obtained in the two investigations on different parameters of relevance (i.e., the respiratory exchange ratio, percentage of peak heart rate, and percentage of peak oxygen uptake at the lactate threshold) (Fig. 2). A moderate degree of heterogeneity was indicated by the $I^{2}$ value of $43 \%$.

\section{HIIT Versus All Other Interventions Except SSG}

One article reported that in comparison to all control interventions except SSG, HIIT produced a large positive mean effect on running economy (mean $d_{\mathrm{ppc} 2} 0.88 \pm$ 0.83; range 0.10-1.64) [13] (Fig. 3).

\section{HIIT Versus SSG}

One comparison of HIIT to SSG revealed a trivial positive mean effect of the former on running economy (mean $d_{\text {ppc2 }} 0.05 \pm 0.05$; range 0.00-0.10) [28] (Fig. 4).

\section{Sprinting Performance}

\section{HIIT Versus All Other Interventions}

HIIT exerted a small positive effect on linear sprinting performance (mean $d_{\mathrm{ppc} 2} 0.10 \pm 0.12$; range $-0.01-0.35$ ) over the various distances $(5,10,20,30$, and $40 \mathrm{~m})$ examined in five different studies. Trivial effects were found for $5 \mathrm{~m}$ [11], $20 \mathrm{~m}$ [10, 11, 30], $30 \mathrm{~m}$ [10], and $40 \mathrm{~m}$ [10]; small positive effects for $10 \mathrm{~m} \mathrm{[13,29],} 30 \mathrm{~m}$ [29], and $40 \mathrm{~m}$ [13]; and one moderate positive effect for $5 \mathrm{~m}$ [29] (Fig. 2). The $I^{2}$ value of $12 \%$ indicated low heterogeneity.

\section{HIIT Versus All Other Interventions Except SSG}

In comparison to all other interventions except SSG, HIIT exerted a trivial positive effect on sprinting performance (mean $d_{\mathrm{ppc} 2} 0.06 \pm 0.08$; range $-0.01-0.17$ ). One small positive effect was found for $10 \mathrm{~m}$ [13], two trivial effects for $20 \mathrm{~m}$ [10, 30], and one trivial effect each for $30 \mathrm{~m} \mathrm{[10]}$ and $40 \mathrm{~m}$ [10] (Fig. 3).

\section{HIIT Versus SSG}

In comparison to SSG, HIIT produced a small positive effect on sprint performance (mean $d_{\mathrm{ppc} 2} 0.15 \pm 0.15$; range 0.01-0.35), with one trivial and one moderate positive effect for $5 \mathrm{~m}[11,29]$, one small positive effect for $10 \mathrm{~m}$ and $30 \mathrm{~m}$ [29], and one trivial positive effect for $20 \mathrm{~m}$ [11, 29] (Fig. 4).

\section{Jumping Performance \\ HIIT Versus All Control Interventions}

In comparison to all other interventions, HIIT had a trivial positive effect on jumping performance (countermovement jump, drop jump, squat jump, and vertical jump) (mean $d_{\mathrm{ppc} 2} 0.01 \pm 0.15$; range $-0.14-0.28$ ). Small negative effects were found in three studies [13, 29, 30], and trivial effects were detected in two cases for the countermovement jump $[4,10]$ and one case each for the drop jump and squat jump [10]. Small positive effects were calculated for the countermovement jump in one investigation [27] and for the drop jump in two studies [4, 29] (Fig. 2). No heterogeneity was observed $\left(I^{2}=0 \%\right)$.

\section{HIIT Versus All Other Interventions Except SSG}

In comparison to all other interventions except SSG, HIIT showed a trivial negative effect on jumping performance (mean $d_{\mathrm{ppc} 2}-0.02 \pm 0.12$; range $-0.14-0.22$ ), based on two small negative effects on the vertical jump $[13,30]$; four trivial effects, two for the countermovement jump $[4,10]$ and one each for the drop jump and squat jump [10]; and one small positive effect for the drop jump [4] (Fig. 3).

\section{HIIT Versus SSG}

In comparison to SSG, HIIT had a trivial positive effect on jumping performance (mean $d_{\mathrm{ppc} 2} 0.08 \pm 0.21$; range -0.13-0.28), as a result of two small positive effects, one on the countermovement jump [27] and one on the drop jump [29], as well as one small negative effect on the countermovement jump [29] (Fig. 4). 


\section{Repeated Sprint Ability HIIT Versus SSG}

Comparison of the impact of HIIT on repeated-sprint ability to that of SSG in one study involving a $12 \times 20-\mathrm{m}$ test [11] resulted in a trivial negative effect $\left(d_{\mathrm{ppc} 2}-0.05\right)$ (Figs. 2 and 4).

\section{Change of Direction Performance HIIT Versus SSG}

One publication showed that in comparison to SSG, HIIT exerted a large positive effect on change-of-direction performance $\left(d_{\mathrm{ppc} 2}\right.$ 0.59) [29] (Figs. 2 and 4$)$.

\section{Soccer-Related Performance Tests HIIT Versus All Other Interventions}

The effect of HIIT on variables related to soccer performance was moderately positive in comparison to that of all other interventions (mean $d_{\mathrm{ppc} 2} 0.42 \pm 0.42$; range $-0.21-1.15$ ) [12, 13, 28] (Fig. 2). The tests involved in this context included technical tests [12, 13, 28], kicking velocity [13], and data collected during actual soccer matches [13] (distance covered, number of sprints, number of contacts with the ball, number of successful and unsuccessful passes). The calculated $I^{2}$ of $8 \%$ indicated a low degree of heterogeneity.

\section{HIIT Versus All Other Interventions Except SSG}

Comparison of HIIT to all other interventions except SSG revealed a moderate positive effect of the former on soccer-related performance, including kicking velocity [13], the Loughborough Soccer Passing Test (LSPT) [12], and data collected during actual soccer matches [13] (mean $d_{\mathrm{ppc} 2} 0.47 \pm 0.39$; range $-0.09-1.15$ ) [12, 13] (Fig. 3).

\section{HIIT Versus SSG}

In comparison to SSG, HIIT exhibited a small negative effect on soccer-related performance $\left(d_{\mathrm{ppc} 2}-0.21\right)$, as assessed by the Ekblom's test [28] (Fig. 4).

\section{Time Efficiency}

The average durations of one session of HIIT and of all the other programs were $33 \pm 14 \mathrm{~min}$ and $41 \pm 15 \mathrm{~min}$, respectively - a difference that is noteworthy, even if not statistically significant. Considered separately, in the two studies involving high-volume endurance exercise as the control training $[4,10]$, the average session lasted significantly longer than in the case of HIIT ( $52 \pm 7$ min vs 31 $\pm 3 \mathrm{~min}$ ). In four studies, the duration of intervention and control sessions was matched [11-13, 27].

\section{Discussion}

The present meta-analysis compares the effects of HIIT on youth soccer players to those of alternative training regimes, including SSG.

The overall findings were as follows:

(i) In comparison to all other interventions, HIIT induces moderate-to-large positive effects on maximal or peak oxygen uptake, variables related to running performance (i.e., maximal running performance, running performance at the lactate threshold and running economy), change-ofdirection performance, and soccer-related performance tests (i.e., technical exercises with the ball and game-specific parameters such as the total distance covered, number of sprints, and number of involvements with the ball)

(ii) In comparison to all other interventions except SSG, HIIT demonstrates moderate-to-large effects on maximal or peak oxygen uptake, variables related to running performance, and, again, soccer-related performance

(iii) In comparison to SSG, HIIT exerts a large effect on change-of-direction ability

(iv) In comparison to all other interventions including or excluding SSG, as well as to SSG alone, HIIT has little or no impact on sprint running performance, jumping performance, or repeated sprint ability

Although endurance performance is unquestionably a key determinant of the success of advanced youth soccer players $[13,32]$, numerous other variables that require time to develop, such as technical and tactical skills, also play major roles $[33,34]$. In this context, time-efficient training in the form of HIIT and SSG, especially in comparison to traditional high-volume training, may offer an excellent approach to improving the endurance performance of these athletes. In this regards, HIIT not only takes less time, but also improves $\mathrm{VO}_{2 \text { peak }}$ to a greater extent than other training strategies [35]. Furthermore, intense regimes of this sort involve physiological loads comparable to those encountered in an actual soccer game, where the heart rate averages $85 \%$ of $\mathrm{HR}_{\max }$ and intensities as high as $90-95 \%$ of $\mathrm{HR}_{\max }$ can be reached $[2,36]$.

In this regards, HIIT and SSG improve endurance parameters (e.g., maximal oxygen uptake, maximal running performance, running performance at the lactate threshold, and running economy) to a similar extent, with slightly higher values of $\mathrm{VO}_{2 \text { peak }}$ for HIIT, in agreement with previous findings [35]. However, SSG also includes soccer-specific drills with the ball or tactical training [14], improving additional determinants of soccer success [19]. Unfortunately, our analysis does not allow definitive determination of which HIIT protocol (sprint 
training in intervals lasting $15 \mathrm{~s}$ to $4 \mathrm{~min}$ ) and SSG (variations in duration, pitch size, and number of players) are most beneficial to youth soccer players. It is noteworthy that there has been some concern that HIIT may be unpleasant for young athletes [37], as well as may lead to overtraining $[38,39]$. Although none of the studies analyzed here reported any signs of overreaching or overtraining, further investigation of the long-term effects of HIIT and SSG is warranted.

The present analysis indicates that improvements in neuromuscular parameters (i.e., sprinting and jumping) with HIIT and SSG were trivial or, at best, small. In most cases, HIIT involved 4-min intervals at $90-95 \%$ of peak heart rate, but not sprinting for only a few seconds at $100 \%$ intensity [40], and the same was true for SSG. In this context, the most pronounced positive effects on sprinting and jumping performance were found in two studies using short (15-30s) intervals of HIIT [4, 29], indicating that for improving sprinting, intervals less than approximately $15 \mathrm{~s}$ may be more beneficial. With the constantly increasing demands on the sprinting ability of youth soccer players from under 13 to younger than 18 years of age [41], the development of explosive strength, often decisive for success, appears to be one major aspect for further development. As also indicated by the effects of HIIT and SSG concerning sprinting and jumping described here, additional training of these abilities appears to be beneficial for youth soccer players. Furthermore, when developing neuromuscular abilities, the state of the child's maturation should be taken into consideration and before puberty, development of strength and speed should be the main target [42]. In adolescents, additional components such as power and hypertrophy should be developed [42].

The single investigation that examined repeated sprints [11] observed a trivial effect of HIIT in comparison to SSG, i.e., SSG and HIIT appear to improve this ability to a similar extent. From a practical point of view, shorter intervals of HIIT or SSG at intensities close to that of repeated sprints may be sufficient to improve such performance. In the one investigation on change-of-direction performance [29], HIIT had a large positive effect in comparison to SSG, in contrast to other findings of greater improvement of this sort with SSG [43], a discrepancy that cannot be explained at present. The effects on repeated sprint ability and change of direction performance both require further scientific evaluation.

In practice, coaches may focus on the effects of various training regimes on performance in connection with soccer-related tests. The medium-positive effect of HIIT on such performance compared to all other interventions can be attributed to the large positive effects on the number of sprints, total distance covered, and number of involvements with the ball, all of which require the endurance that can be achieved with HIIT. The negative effect of HIIT in the Ekblom's test in comparison to SSG (Fig. 4) may be explained by the various soccer-specific movements involved in this test (i.e., jumping, slalom running, running backward, changes in direction, running sideways), which are most closely mimicked in SSG.

HIIT and SSG provide similar benefits with respect to most of the parameters analyzed here (Fig. 4). Moreover, SSG may offer additional advantages, improving essential neuro-muscular and cognitive skills such as reaction time, decision-making, and change-of-direction speed [44]. Participants may also experience greater motivation [45] and enjoyment [27] when performing SSG than HIIT protocols that are less sport-specific. At the same time, the intensity of SSG can be varied greatly, being higher on larger pitches and with a smaller number of players [16], and the optimal design remains to be determined.

From a practical point of view, the choice of a HIIT or SSG protocol for training youth soccer players depends to a large extent on the time-point during the season and the coach's overall strategy. HIIT induces somewhat more pronounced increases in $\mathrm{VO}_{2 \text { peak }}$ than SSG, thereby developing adequate aerobic endurance. During a season, running-based protocols that do not involve ball drills often appear impractical. From this perspective, SSG may provide a viable alternative for maintaining endurance and skills such as ball handling and tactical thinking.

\section{Limitations}

The following limitations of the present analysis need to be considered: (1) Only nine studies met our criteria for inclusion, and in some cases, no more than one ES could be calculated for each parameter; (2) for some parameters, there was considerable heterogeneity (as indicated by the $95 \% \mathrm{CI}$ ), especially for running performance at the lactate threshold; (3) we categorized each report on the basis of what the authors stated. For instance, in one of the studies [11] involving repeated sprints as the intervention, the authors stated explicitly that these were performed as a form of HIIT; (4) we cannot assess the benefits of HIIT and SSG to youth soccer players in individual competitive situations, since a standardized soccer match (or simulation thereof) does not yet exist; (5) there may be major age-dependent differences in the development of the parameters investigated here, and there is no clear-cut division between youth and adult soccer players; (6) although all of the HIIT and SSG protocols analyzed met our criteria, these protocols were somewhat heterogeneous, especially with respect to duration, intensity, and ratio of load to rest (Table 2). 


\section{Conclusions}

On the basis of the present analysis, we conclude that HIIT in general and the more sport-specific SSG both result in similar improvements in maximal or peak oxygen uptake, variables related to running performance (i.e., maximal running performance, running performance at the lactate threshold, and running economy), and soccer-related performance tests in youth players. In contrast, repeated sprint ability and sprinting and jumping performance are virtually unaffected by either HIIT or SSG. Change of direction performance was improved to a greater extent by HIIT than SSG, but this difference requires further investigation. Thus, HIIT and SSG both offer an effective approach to simultaneously improve certain physiological characteristics and the sport-specific skills of youth soccer players.

\section{Abbreviations \\ AR: Active rest; Cl: Confidence interval; CinD: Change-in-direction run; CMJ: Countermovement jump; DJ: Drop jump; ES: Effect size; HIIT: High- intensity interval training; $H R_{\text {max }}$ : Maximal heart rate; $H_{\text {peak: }}$ Peak heart rate; HVT: High-volume training; IAT: Individual anaerobic threshold; \\ Lac max: Maximal blood lactate concentration; LIST: Loughborough Intermittent Shuttle Test; LSPT: Loughborough Soccer Passing Test; LT: Lactate threshold; MAS: Maximal aerobic speed; MSFT: Multistage fitness test; n.i.: Not indicated; PEDro: Physiotherapy Evidence Database; PR: Passive rest; RE: Running economy; Reps: Repetitions; RSA: Repeated sprint ability; SJ: Squat jump; SSG: Small-sided game; TT: Technical training; TTE: Time to exhaustion; VJ: Vertical jump; $V_{\text {max/peak: Maximal velocity during incremental }}$ step test; $\mathrm{VO}_{2 \text { max }}$ : Maximal oxygen uptake; $\mathrm{VO}_{2 \text { peak: }}$ Peak oxygen uptake; VOT: Volume-oriented training at low intensities; $\mathrm{VT}$ : Ventilatory threshold; YYIETL1: Yo-Yo intermittent endurance test level 1; YYIRTL1: Yo-YO intermittent recovery test level 1}

\section{Acknowledgements}

Not applicable.

\section{Funding}

This publication was funded by the German Research Foundation (DFG) and the University of Wuerzburg in the funding programme Open Access Publishing.

\section{Availability of Data and Materials}

Data sharing not applicable as no datasets were generated or analysed during the present study.

\section{Authors' Contributions}

PK drafted the manuscript. $\mathrm{FAE}, \mathrm{H}-\mathrm{CH}$, and $\mathrm{BS}$ read and approved the final manuscript. All authors read and approved the final manuscript.

\section{Ethics Approval and Consent to Participate}

Not applicable.

\section{Consent for Publication}

Not applicable.

\section{Competing Interests}

The authors, Philipp Kunz, Florian Azad Engel, Hans-Christer Holmberg, and Billy Sperlich, declare that they have no competing interests.

\section{Publisher's Note}

Springer Nature remains neutral with regard to jurisdictional claims in published maps and institutional affiliations.

\section{Author details}

'Integrative \& Experimental Exercise Science and Training, Institute of Sport Science, University of Würzburg, Judenbühlweg 11, 97082 Würzburg, Germany. ${ }^{2}$ Institute of Sport and Sport Science, Heidelberg University, Heidelberg, Germany. ${ }^{3}$ School of Sport Sciences, UiT The Arctic University of Norway, Tromsø, Norway. ${ }^{4}$ Swedish Winter Sports Research Centre,

Department of Health Sciences, Mid Sweden University, Östersund, Sweden.

Received: 31 July 2018 Accepted: 11 February 2019

Published online: 21 February 2019

\section{References}

1. Stølen T, et al. Physiology of soccer: an update. Sports Med. 2005;35(6): 501-36.

2. Mendez-Villanueva A, et al. Match play intensity distribution in youth soccer. Int J Sports Med. 2013;34(2):101-10

3. Vanderford ML, et al. Physiological and sport-specific skill response of olympic youth soccer athletes. J Strength Cond Res. 2004;18(2):334-42.

4. Faude $\mathrm{O}$, et al. High intensity interval training vs. high-volume running training during pre-season conditioning in high-level youth football: a cross-over trial. J Sports Sci. 2013;31(13):1441-50.

5. Meckel $Y$, et al. Effects of a very short-term preseason training procedure on the fitness of soccer players. J Sports Med Phys Fitness. 2014;54(4):432-40.

6. Gibala MJ, et al. Physiological adaptations to low-volume, high-intensity interval training in health and disease. J Physiol. 2012;590(5):1077-84.

7. Rampinini E, et al. Factors influencing physiological responses to small-sided soccer games. J Sports Sci. 2007;25(6):659-66.

8. McMillan K, et al. Physiological adaptations to soccer specific endurance training in professional youth soccer players. Br J Sports Med. 2005;39(5): 273-7.

9. Impellizzeri FM, Rampinini E, Marcora SM. Physiological assessment of aerobic training in soccer. J Sports Sci. 2005;23(6):583-92.

10. Sperlich B, et al. Effects of 5 weeks of high-intensity interval training vs. volume training in 14-year-old soccer players. J Strength Cond Res. 2011; 25(5):1271-8.

11. Hill-Haas SV, et al. Generic versus small-sided game training in soccer. Int J Sports Med. 2009:30(9):636-42.

12. Impellizzeri FM, et al. Effects of aerobic training on the exercise-induced decline in short-passing ability in junior soccer players. Appl Physiol Nutr Metab. 2008;33(6):1192-8.

13. Helgerud J, et al. Aerobic endurance training improves soccer performance. Med Sci Sports Exerc. 2001;33(11):1925-31.

14. Hill-Haas SV, et al. Physiology of small-sided games training in football: a systematic review. Sports Med. 2011:41(3):199-220.

15. Maclnnis MJ, Gibala MJ. Physiological adaptations to interval training and the role of exercise intensity. J Physiol. 2017:595(9):2915-30.

16. Halouani J, et al. Small-sided games in team sports training: a brief review. J Strength Cond Res. 2014:28(12):3594-618.

17. Gibala MJ, et al. Short-term sprint interval versus traditional endurance training: similar initial adaptations in human skeletal muscle and exercise performance. J Physiol. 2006;575(Pt 3):901-11.

18. Esfarjani F, Laursen PB. Manipulating high-intensity interval training: effects on VO2max, the lactate threshold and $3000 \mathrm{~m}$ running performance in moderately trained males. J Sci Med Sport. 2007;10(1):27-35.

19. Sarmento $\mathrm{H}$, et al. Talent identification and development in male football: a systematic review. Sports Med. 2018;48(4):907-31.

20. Liberati A, et al. The PRISMA statement for reporting systematic reviews and meta-analyses of studies that evaluate healthcare interventions: explanation and elaboration. BMJ. 2009:339:b2700.

21. Buchheit M, Laursen PB. High-intensity interval training, solutions to the programming puzzle. Part II: anaerobic energy, neuromuscular load and practical applications. Sports Med. 2013;43(10):927-54.

22. Laursen PB, Jenkins DG. The scientific basis for high-intensity interval training: optimising training programmes and maximising performance in highly trained endurance athletes. Sports Med. 2002;32(1):53-73.

23. Olivo SA, et al. Scales to assess the quality of randomized controlled trials: a systematic review. Phys Ther. 2008;88(2):156-75.

24. Carlson KD, Schmidt FL. Impact of experimental design on effect size: findings from the research literature on training. J Appl Psychol. 1999;84(6):851.

25. Morris SB. Estimating effect sizes from pretest-posttest-control group designs. ORM. 2008;11(2):364-86. 
26. Fröhlich $M$, et al. Outcome effects and effects sizes in sport sciences. Int J Sports Sci Eng. 2009;3(3):175-9.

27. Los Arcos A, et al. Effects of small-sided games vs. interval training in aerobic fitness and physical enjoyment in young elite soccer players. PLoS One. 2015;10(9):e0137224.

28. Impellizzeri FM, et al. Physiological and performance effects of generic versus specific aerobic training in soccer players. Int I Sports Med. 2006; 27(6):483-92.

29. Faude $O$, et al. The effect of short-term interval training during the competitive season on physical fitness and signs of fatigue: a crossover trial in high-level youth football players. Int I Sports Physiol Perform. 2014;9(6): 936-44.

30. Siegler J, Gaskill S, Ruby B. Changes evaluated in soccer-specific power endurance either with or without a 10-week, in-season, intermittent, highintensity training protocol. J Strength Cond Res. 2003:17(2):379-87.

31. Stegmann H, Kindermann W, Schnabel A. Lactate kinetics and individual anaerobic threshold. Int J Sports Med. 1981;2(3):160-5.

32. Hoff J, Helgerud J. Endurance and strength training for soccer players: physiological considerations. Sports Med. 2004;34(3):165-80.

33. Memmert D. Testing of tactical performance in youth elite soccer. J Sports Sci Med. 2010;9(2):199-205.

34. Kannekens R, Elferink-Gemser MT, Visscher C. Positioning and deciding: key factors for talent development in soccer. Scand J Med Sci Sports. 2011;21(6): 846-52

35. Engel FA, et al. High-intensity interval training performed by young athletes: a systematic review and meta-analysis. Front Physiol. 2018:9:1012.

36. Rebelo A, et al. Physical match performance of youth football players in relation to physical capacity. Eur J Sport Sci. 2014;14(Suppl 1):S148-56.

37. Fernandez-Fernandez J, et al. The effects of sport-specific drills training or high-intensity interval training in young tennis players. Int I Sports Physiol Perform. 2017:12(1):90-8.

38. Kentta G, Hassmen P, Raglin JS. Training practices and overtraining syndrome in Swedish age-group athletes. Int J Sports Med. 2001;22(6):460-5.

39. Winsley R, Matos N. Overtraining and elite young athletes. Med Sport Sci. 2011;56:97-105

40. Lockie RG, et al. The effects of different speed training protocols on sprint acceleration kinematics and muscle strength and power in field sport athletes. J Strength Cond Res. 2012;26(6):1539-50.

41. Buchheit $\mathrm{M}$, et al. Match running performance and fitness in youth soccer. Int J Sports Med. 2010;31(11):818-25.

42. Lloyd RS, Oliver JL. The youth physical development model: a new approach to long-term athletic development. J Strength Cond Res. 2012; 34(3):61-72.

43. Chaouachi A, et al. Multidirectional sprints and small-sided games training effect on agility and change of direction abilities in youth soccer. J Strength Cond Res. 2014;28(11):3121-7.

44. Young $W$, Rogers $N$. Effects of small-sided game and change-of-direction training on reactive agility and change-of-direction speed. J Sports Sci. 2014;32(4):307-14.

45. Buchheit $\mathrm{M}$, et al. Game-based training in young elite handball players. Int J Sports Med. 2009;30(4):251-8.

\section{Submit your manuscript to a SpringerOpen ${ }^{\circ}$ journal and benefit from:}

- Convenient online submission

- Rigorous peer review

- Open access: articles freely available online

- High visibility within the field

- Retaining the copyright to your article

Submit your next manuscript at $\boldsymbol{\nabla}$ springeropen.com 